\title{
The Internet and the Physician-Patient Relationship
}

\author{
Randale C. Sechrest MD
}

Published online: 24 June 2010

(C) The Association of Bone and Joint Surgeons ( 2010

\begin{abstract}
Background Since the emergence of the public Internet in the early 1990s, the healthcare industry has been struggling to understand how best to utilize this resource. During the last decade there has been an increase in both the interest and participation by healthcare providers in the Internet space, but many observers continue to push for more development of healthcare resources to better support the provider-patient relationship.

Questions/purposes This paper will review the historical development of the Internet, the core concepts that have driven the emergence and evolution of the Internet as a mass medium of information exchange, and how the healthcare industry can harness the Internet to improve the provider patient relationship.

Where are we now? The healthcare industry continues to lag behind other industries that have been transformed by the Internet. Numerous industries including travel, real estate, retail sales, and banking have migrated both comprehensive information resources and transactions to the Internet in order to improve efficiency and customer satisfaction. That same process is occurring now in the healthcare industry. Credible and comprehensive Information resources are beginning to mature. Transactions are still in their infancy, reflecting a continued concern about privacy and security.

Where do we need to go? We need to improve information resources to educate and inform patients. Improving
\end{abstract}

Dr. Sechrest is the owner and CEO of Medical Multimedia Group.

R. C. Sechrest $(\square)$

Medical Multimedia Group, LLC, 2300 Regent Street, Suite 205,

Missoula, MT 59801, USA

e-mail: randale@sechrest.com the availability and credibility of information resources will empower patients to make better healthcare decisions and I contend will ultimately reduce the cost of delivering care.

How do we get there? Orthopaedists must first recognize the value of information resources to the patient. Effective communication with patients is a critical component of providing healthcare services. All healthcare providers should reflect on the importance of developing an effective communications strategy for their own practice and consider the benefits of participating in efforts by professional organizations to improve existing information resources.

\section{Introduction}

The Internet is the dominant medium for obtaining information in 2010. This is not restricted to specific types of information; essentially all types of information are collapsing into a paradigm that uses the Internet as the primary distribution mechanism. There are many new and novel technological developments that differentiate networked information from what has come before, but the paradigm shift toward the Internet is characterized by one key attribute: the information is constantly available for random access by the end user. Within the last decade and a half, this transition has occurred for all forms of information, including such mundane information as weather, restaurant menus, and bus schedules, to more elaborate forms of information such as music, movies, and education. To make the decisions driving our lives today, we plug into a virtual space, travel that space to locate resources, and either execute a transaction online or obtain the necessary information to execute a transaction in the real world. 
Health care has been somewhat slow to embrace this paradigm shift. But now, driven by the ubiquity of the Internet and its impact on all aspects of life, there is really no choice; the healthcare industry is forced to catch up. Healthcare providers are recognizing the importance of this shift in how we search for and obtain information. As a result, they are poised for better understanding the value and importance of the "information component" as an integral part of the healthcare service. Providing relevant information is important and demands competency and skill, just as diagnosis and treatment do.

This article will provide an overview of the core concepts that have driven development of the Internet to this point, identify the key areas of the healthcare Internet that are in transition, and recommend areas for further investigation and development. The primary task at hand is to increase awareness among providers of the role highquality information services play in delivering high-quality health services and begin improving the availability, accessibility, and quality of the information available.

\section{Where are we now?}

In the United States, $74 \%$ of the adult population now has access to the Internet [4] and $63 \%$ of adults have broadband connections at home [6]. Mobile information through the cellular phone system is growing dramatically [7]. Sixtyone per cent of adults use the Internet to gather healthrelated information [4]. The Internet is becoming the initial point of contact for patients who are trying to understand health issues and treatment options. The supply side of health information is in transition as well. Internet publishing has shifted from tightly controlled editorial processes symbolized by the "portal" to the chaotic syndication model of social media. The type of content being created has shifted from primarily textual content to rich multimedia as broadband connections have become more prevalent. The healthcare Internet is poised to enter a new phase where the agenda may shift from a focus dominated by marketing to one promising more empowerment for patients and the ability to execute meaningful healthcare transactions.

A paradigm shift in communication has occurred during the last two decades and continues to evolve. According to the most recent data available (October 2009) from the Pew Research Center's Internet and American Life Project [4], $74 \%$ of American adults use the Internet. In 1995, only $13 \%$ could be said to access the Internet in some form [4]. This represents the fastest rate of adoption of any medium of communication in history.

The "always-on" broadband connection has overcome two barriers that once stood in the way of making networked information truly useful to the majority of home users-inconvenience and speed. Before the persistent broadband connection became the norm, the time required to make a connection to the Internet meant traditional information sources still trumped the network as the default resource; looking something up in a book, a magazine, or the Yellow Pages was preferable. Likewise, once the connection was initiated, the time required to download multimedia information created a further disincentive. As of April 2009, home broadband adoption stood at $63 \%$ of adult Americans, up from 55\% in May 2008 [6]. To put this in perspective, one should note, in 2000, home broadband adoption stood at 4\% [6]. The clunky, slow dial-up connection that characterized the Internet experience in the 1990s has given way to instant access and near real-time transfer of much of the media we consume.

There is an anticipation this pattern of adoption will be repeated within the mobile space. Today, 39\% of American adults routinely use a cellular device to connect to the Internet [7]. Many companies that are leaders in the computer and Internet industry have migrated to the mobile marketplace, such as Google ${ }^{\mathrm{TM}}$ with the Android $^{\mathrm{TM}}$ Mobile Operating System and Apple ${ }^{\circledR}$ with the iPhone ${ }^{\circledR}$. Perhaps the take-home message is that it will be increasingly irrelevant to consider the cellular system as distinct from the Internet.

Today, if you are a provider of goods or services and do not participate in the Internet, increasingly you do not exist for a growing part of the population. Likewise, consumers who choose not to participate in the services afforded by the Internet are finding themselves at a disadvantage. Internet illiteracy is not necessarily benign; there is a cost to the resulting exclusion. Not too long ago, it was not unusual to hear many physicians proudly proclaim they did not use computers and were not about to start. Having a secretary print off an e-mail for the physician to first read and then dictate a response followed the traditional method of correspondence and communication familiar to this generation. Researching the medical literature meant a trip to the medical library to browse through volume after volume of Index Medicus. There were very few penalties to be paid if one did not have the skills to utilize a computer or understand the nuances of the online world. That situation has changed dramatically in less than a decade.

It is not that people without Internet access simply lack convenience; they are actually at a disadvantage in terms of the depth and breadth of information available. As more resources move online, there is less access to offline resources. And it is not simply that the traditional resources of information are layering on an Internet component. In many cases, entirely new information resources are appearing that have no offline equivalent to the resources originating online. For example, consider what is occurring 
now with the demise of the newspaper and print magazine industry. Many of these resources are moving online and either reducing or altogether eliminating offline distribution. While convenience and availability of online access continue to improve the efficiency of information access, the cost of utilizing offline resources in terms of time and money is stagnant at best and most likely will continue to rise. As a consumer of goods or services, if you do not utilize the Internet, you are increasingly finding yourself at a disadvantage with less access to the information necessary to optimally satisfy day-to-day needs.

The physical world and the virtual world are becoming interdependent. Every product or service has a physical component and an information component. The physical component of a product is relatively easy to define and understand as the physical artifact, for example, an automobile. The physical component of a service is a bit more complex but is perhaps best understood as an action occurring between two entities that requires some type of active participation from both parties. For our purposes, this is best illustrated by a surgical procedure.

By contrast, information components are somewhat ethereal; they are made up of all the various bits of information available to help you locate, choose, access, and utilize a product or service. Information components become important in two ways: (1) they help us choose what products to acquire or services to access and (2) they help us better utilize the product or service after the transaction is complete. A set of rich, robust information components wraps around the actual product or service and increases the value of a physical product or service.

The Internet has created a virtual space that makes finding and accessing the information component of products and services nearly effortless. The incredible power of search engines layered upon an explosion of network accessible information has expanded our capabilities for organizing and retrieving information. The Internet has also vastly amplified the ability to separate the information component and the physical component and deliver the information component online-on a self-serve, just-intime basis. Finally, the Internet has created a new marketplace where the information components themselves have become profitable products and services. Consider, for example, a product such as an eBook or movie and a service such as voicemail or a second opinion by a radiologist. Information products have been profoundly impacted by the Internet as our entire concept of the marketplace has evolved. Clearly there are "products" such as medications and medical devices and "services" such as MRI scans and surgical procedures requiring an individual physically access a healthcare provider to obtain that product or service. But, there is a nearly infinite array of information components wrapping around those products and services.
The way we locate and access information has changed. The "search" paradigm has achieved dominance in the pattern of information retrieval of the Internet. Directory structures, while not completely moribund, have proven less compelling for initial information retrieval when compared to search engines. Much of the information created and distributed today is syndicated outward rather than controlled within a fixed directory hierarchy. What this means is the quality of the metadata attached to an information asset becomes much more important in terms of making the information discoverable. Those metadata are also important for pointing back to the source of the information, thus conveying attribution, credibility, and legitimacy to the asset. Control has given over to a form of "controlled chaos."

Search engines serve as brokers of information rather than creators of information. In just a few short years, Google ${ }^{\mathrm{TM}}$ has managed to illustrate: "Information access has greater market value than information creation" [1]. Abelson et al. [1] base this observation on the fact that the market capitalization of Google $\mathrm{e}^{\mathrm{TM}}$ (\$175 billion) exceeds that of many publishers (capitalization data updated as of November 2009) such as the New York Times ( $\$ 1$ billion), Time Warner ( $\$ 36$ billion), Disney ( $\$ 53$ billion), and News Corp ( $\$ 32$ billion). The ability to organize and find the right information at the right time appears to be extremely valuable in driving all different types of transactions.

The devices we use to interface with information continue to change and become more robust. The personal computer has evolved to become a multimedia workstation that can not only display multimedia content but also produce a wide array of multimedia content at a fraction of the previous cost. The mobile phone is not far behind the computer in the capabilities to access the network and in some instances produce content. The increasing penetration of broadband connections has enabled the Internet to evolve into a vast, robust distribution system for rich multimedia content. The falling cost of production for multimedia information coupled with the increasing availability of broadband access is collapsing all communications — voice, text, image, and video-onto the digital Internet platform.

The key attributes defining communication in the Internet age are current, accurate, and accessible. "Current" has taken on an entirely new meaning with the ascendance of the Internet as the primary source of information gathering. Compared to the Internet, the newspaper seems sluggish. The old TV news phrase "film at 11" seems ridiculous to even contemplate when video can be uploaded to YouTube immediately from a cell phone. The notion of what is current has been compressed substantially, but the question remains as to whether that gain in speed has been detrimental to accuracy. 
Our concept of authority is evolving. There continues to be an ongoing debate on whether the "wisdom of crowds" trumps expert opinion. The proponents of Wikipedia ${ }^{\circledR}$ and other prominent examples of "crowd sourcing" essentially argue accuracy is an emergent property that can be expected to refine itself over time based on the large numbers of contributors it is possible to aggregate on the Internet [10]. Others [8] have argued we are seeing a devaluing of expertise that will eventually lead to a degradation of actual knowledge and credibility. This experiment continues to progress and the result is far from certain.

\section{Where do we need to go?}

To effectively and efficiently manage health and deal with health issues, individuals need more than access to healthrelated products and physical services. They also need to know "what to do" and "how to do it." To take action to deal with a healthcare problem starts with a series of questions: What is the nature of the problem? What do I need to do to correct or manage the problem? What services do I need to access to take action? What services exist to assist me in managing this problem? How do I access those resources?

Think about the "information components" hidden in these tasks associated with dealing with a healthcare issue. The answers to these questions represent the information components of healthcare that wrap around the actual service being provided and increase the value of the service. Increasingly, as the time devoted to actual face-toface contact between patient and provider diminishes in an effort to increase productivity, the information component of a healthcare transaction is less likely to be delivered by the provider. In the overall delivery of care, providing information simply has a lower priority than performing a procedure requiring the unique expertise of the provider.

The current approach dominating the healthcare transaction today is to physically present oneself to the system to complete nearly all of the tasks associated with understanding "what to do" and "how to do it." This is inefficient for both patient and provider and needlessly expensive.

Contrast that to the industries that have been transformed by the Internet. Today, literally no one begins at the automobile dealership to purchase a car, nor do they rely on the salesman as the sole conduit for the information wrapped into buying and maintaining a car. The same can be said for nearly any product or service. Industries as diverse as banking, automobile sales, stock trading, real estate, and travel planning have moved much of the information needed to both choose to purchase and utilize their products and services to the Internet where it is provided "self-serve" for customers 24 hours a day. By doing this, these industries have increased efficiency and reduced cost-for both their customers and themselves-and customers are actually more satisfied with the relationship.

We should distinguish between transactions and information components. Transactions are more complicated and up the stakes in terms of security and privacy. One of the main reasons health care has been slow to embrace the Internet revolution is about the risk of transactions, not information components. But, the information component enables transactions. The information component represents actionable information that convinces an individual to make the decision to execute a transaction.

Effective communication is as much about accessibility as it is about information content. Accessibility in the context of the Internet incorporates several attributes: understandable, findable, and reachable. Information intended to serve a specific audience must be presented in such a way as to be understandable to that audience. Communicating complex information, such as healthcare information, from provider to patient has always represented a major challenge. In the past, that usually meant crafting textual information at the appropriate reading level to maximize the potential for understanding on the part of the patient. In today's communication environment, textual content merely serves as the initial "storyboard" for creating the derivative multimedia. The increasing ease of creating and merging multiple types of multimedia assets-textual, visual, and auditory-to develop a multimedia narrative that communicates the message is becoming the norm. The end result may be the message is distributed in a variety of formats, including tweet, e-mail, blog, video, screencast, and podcast, to name a few.

Information published on the Internet must be visible to the seeker. The dominance of search as the driving force for information gathering means a considerable amount of attention must be paid to optimizing the metadata surrounding the information to make it more findable by search engines.

Finally, the information asset must be reachable. The Internet is about "just-in-time, self-serve, random access." Information not published in digital format on the Internet is not reachable for all intents and purposes. We are all witnessing an evolution in how we expose ourselves to the world around us, both in our personal lives and in our professional lives. Today, we are more likely to post the digital photographs of our most recent trip to Facebook and share them with a few friends and family rather than go to the trouble of contacting each one individually. We simply make the information available and allow the end user to decide what they find valuable and when they want to access it. The same dynamic works for each bit of information you and your staff discuss with patients each and every day. 
Neither the tools of production nor distribution represent a major barrier to entry for multimedia information creation. The existing barriers are primarily the skills required to utilize the tools and the time and effort expended to create and maintain the assets. The benefits to be gained are the increased efficiency in managing the information components of your practice and the improved communication with your patients. Increasingly, the benefits are beginning to outweigh the costs.

\section{Discussion}

Today, we find ourselves roughly a decade and a half into a transition toward a network-centric paradigm of information distribution characterized as always on, random access, and ubiquitous. We have access to an increasing number of information services that have successfully distinguished the information component from the physical component of a product or service; these information providers understand the relationship of the information component to the physical product or service and how the information component enhances the value of the physical products and services. A profound change, driven by the layering of robust search technologies over a constantly growing volume of information, has placed enormous amounts of information at our fingertips. This information forms a virtual space, a market space, that increasingly forms the mechanism by which we make decisions and execute transactions.

The Internet has reduced the "friction" in executing many types of transactions, but it has truly revolutionized how we locate and access the information necessary to carry on our lives. There are incredible efficiencies yet to be gained in health care by focusing efforts on improving the information available surrounding the delivery of health care. However, the majority of the valuable information distributed each day in the course of providing a healthcare service is either not available or not accessible to the end user in any way other than through a face-to-face interaction with a healthcare provider. In health care, we have been slow to realize there is something of considerable value we can provide our patients that wraps around and enhances the expensive, physical, human-to-human interaction. We should acknowledge the need patients have for credible, understandable, and accessible information that enables healthcare decision making and work to make such information available.

Where do we need to go?

It is necessary for us to recognize and understand this paradigm shift. It may no longer be reasonable to expect patients will continue to see making an appointment and physically accessing healthcare services that are primarily an exchange of information as the only option. In all other aspects of their lives, consumers are able to easily search for, locate, become informed, and begin a relationship with a provider of nearly any type of product or service. They also expect easy access to information allowing them to effectively utilize the good or service after the transaction is complete. It is fast becoming a "self-service" world. As healthcare providers, we need to understand how we should prepare to respond to these expectations.

Orthopaedic surgeons see themselves first and foremost as "doers." We primarily inhabit the world of the "physical" component and tend to perceive the information component of what we do as a nice addition, but not necessarily critical to the final product-the surgical outcome. This is a false dichotomy. As healthcare providers, we need to better understand the difference between the information component and the physical component of the services we provide. We need to recognize the hidden value of the information component and dedicate the same attention to ensuring this aspect of the healthcare service we provide is delivered effectively and appropriately.

The focus of the information also needs to change. There is a big difference between information designed to market an agenda from that designed to empower. Information designed to get patients through the door is primarily marketing driven. Information designed to reduce the need for a costly human-to-human interaction results in empowerment for patients and may increase the efficiency in delivering healthcare services. Much of the Internetbased healthcare information produced by hospitals and physician practices to date is clearly marketing driven. The early days of the healthcare Internet were characterized by a traditional publishing model. Health-related content is simply produced and distributed "one way," with little opportunity for meaningful engagement by the audience. This model was focused on creating and controlling a message in a top-down fashion.

How do we get there?

This is beginning to change as the healthcare Internet sector embraces the Web 2.0 paradigm and transitions toward what has been described as "Health 2.0" [5]. Health 2.0 is characterized by participation. Health 2.0 resources are evolving into software applications that attempt to promote and nurture a user-to-user conversation. Many Health 2.0 resources attempt to pool large amounts of data about specific health issues gleaned from the user community. This information is then shared with the user community to empower healthcare decisions. The impact of these resources is yet to be determined and represents 
fertile ground for further investigation on their effect on costs, quality, and patient satisfaction.

It is clear patients continue to use the Internet to help with medical decision making. In a survey commissioned by the California Healthcare Foundation, 55\% of respondents stated, during the past 12 months, they had consulted a physician for health-related information, while 59\% stated they accessed the Internet for this type of information [9]. It is unlikely this trend will reverse itself. Our challenge as healthcare providers will be to decide how we will participate in the Internet conversation. Each of us will need to decide what we are willing to offer in terms of information resources and communication tools that allow patients to interact with our practice.

It is also clear there is a persistent disconnect between what patients desire in terms of electronic communication with their physician and physicians' willingness to provide this service [2]. This has created a vacuum that is being filled by other parties willing to meet this need in the marketplace. This trend will likely continue until physicians recognize a need to communicate better with patients. Perhaps the problem is best stated by Farley and Weinstein who note: "Clearly, there is a large discrepancy between how orthopaedic surgeons perceive themselves and how other orthopaedic surgeons and patients perceive them as communicators" $[3,11]$. Developing a better understanding of this discrepancy would be a good start in developing the skills, communication tools, and resources to narrow that gap.

\section{References}

1. Abelson H, Ledeen K, Lewis H. Blown to Bits: Your Life, Liberty, and Happiness After the Digital Explosion. 1st ed. Upper Saddle River, NJ: Addison-Wesley Professional; 2008.

2. Biermann JS, Golladay GJ, Peterson RN. Using the Internet to enhance physician-patient communication. $J$ Am Acad Orthop Surg. 2006;14:136-144.

3. Farley FA, Weinstein SL. The case for patient-centered care in orthopaedics. J Am Acad Orthop Surg. 2006;14:447-451.

4. Fox S, Jones S. The social life of health information. Available at: http://www.pewinternet.org/Reports/2009/8-The-Social-Life-ofHealth-Information.aspx. Accessed November 25, 2009.

5. Health 2.0. http://en.wikipedia.org/wiki/Health_2.0. Accessed April 19, 2010.

6. Horrigan J. Home broadband adoption. Available at: http://pew internet.org/Reports/2009/10-Home-Broadband-Adoption-2009. aspx. Accessed November 25, 2009.

7. Horrigan J. The mobile difference. Available at: http://www.pew internet.org/Reports/2009/5-The-Mobile-Difference-Typology. aspx. Accessed November 25, 2009.

8. Keen A. Cult of the Amateur. 1st ed. New York, NY: Bantam Dell Pub Group; 2005.

9. Sarasohn-Kahn J. The wisdom of patients: health care meets online social media. Available at: http://www.chcf.org/topics/ chronicdisease/index.cfm?itemID $=133631$. Accessed November 25, 2009.

10. Tapscott D, Williams AD. Wikinomics: How Mass Collaboration Changes Everything. 1st ed. New York, NY: Penguin Group; 2006.

11. Tongue JR, Epps HR, Forese LL. Communication skills for patient-centered care: research-based, easily learned techniques for medical interviews that benefit orthopaedic surgeons and their patients. J Bone Joint Surg Am. 2005;87:652-658. 